\title{
Global Asymptotic Stabilization of the Attitude and the Angular Rates of an Underactuated Non-Symmetric Rigid $\operatorname{Body}^{\star}$
}

\author{
Daniele Casagrande ${ }^{\mathrm{b}}$, Alessandro Astolfi ${ }^{\mathrm{b}, \mathrm{c}}$, Thomas Parisini ${ }^{\mathrm{a}}$ \\ ${ }^{a}$ Dip. di Elettrotecnica, Elettronica ed Informatica, Università degli Studi di Trieste, via A. Valerio 10, 34127 Trieste, Italy \\ ${ }^{\mathrm{b}}$ Dept. of Electrical and Electronic Engineering, Imperial College London, Exhibition Road, London SW $2 A Z$, UK \\ ${ }^{\mathrm{c}}$ Dip. di Informatica Sistemi e Produzione, Università di Roma "Tor Vergata", Via del Politecnico 1 - 00133 Roma, Italy
}

\begin{abstract}
The paper deals with the global stabilization of both the attitude and the angular velocities of an underactuated rigid body. First a stability theorem is proven for a class of systems; subsequently, the equations describing the physics of the rigid body are presented, showing that the rigid body belongs to the considered class of systems, and a sufficient condition for the application of the theorem to the stability of the rigid body equilibrium is pointed out. Finally, some simulations results are reported showing the effectiveness of the proposed methodology.
\end{abstract}

Key words: Rigid body, switching control, Lyapunov function.

\section{Introduction}

In the past, the problem of asymptotic stabilization of the zero equilibrium of the rigid body attitude has been widely studied. For instance in (Crouch, 1984) necessary and sufficient conditions have been provided for the system to be controllable and, based on this analysis, an algorithm has been devised for which the zero equilibrium of the linearized system is locally stabilized. Byrnes and Isidori (1991) have proven that no continuous stabilizing feedback exists for the underactuated rigid body and they propose a feedback control law that locally asymptotically drives the rigid body to a motion around one of the axes. In (Krishnan et al., 1992), similarly to (Crouch, 1984), available controllability techniques are iteratively applied in a sort of closed loop law. Local asymptotic stability has also been achieved by Morin et al. (1995) by using the Centre Manifold Theorem to derive a smooth time-varying control law and, more in general, by Coron and Keraï (1996).

\footnotetext{
* This work was partially supported by the italian Regione Friuli Venezia Giulia (legge 11/2003).

Email addresses: d.casagrande@ic.ac.uk (Daniele Casagrande), a.astolfi@ic.ac.uk (Alessandro Astolfi), t.parisini@paperplaza.net (Thomas Parisini).
}

Other works focus on the stabilizability of the velocities; in (Astolfi, 1999), for instance, it is proven that a suitable "practical" exponential stability property can be achieved also when not all the states are measurable. As far as the underactuated rigid body is concerned, Aeyels and Szafranski (1988) have shown that a single torque cannot asymptotically stabilize the zero equilibrium, while in (Aeyels, 1985) the simpler problem of practical stabilization is shown to be solvable even if only one torque is available. Furthermore, the case of a symmetric rigid body has been considered by Sontag and Sussmann (1988) and by Outbib and Sallet (1992).

In this paper the simultaneous stabilization of both the attitude and the angular velocities is considered; the proposed solution is based on a novel approach, the main feature of which can be described as follows. Consider a more generic version of the equations describing the physics of the rigid body, i.e. a system constituted by two equations, $\dot{\boldsymbol{\omega}}=\mathbf{F}(\boldsymbol{\omega}, \mathbf{w})$ and $\dot{\boldsymbol{\xi}}=\mathbf{G}(\boldsymbol{\omega}, \boldsymbol{\xi})$, which are referred to as dynamic and kinematic equation, respectively. Suppose that the dynamic equation is completely controllable by means of the input $\mathbf{w}$ while the kinematic equation is not directly affected by the input and is, instead, affected by the dynamic state variable $\boldsymbol{\omega}$. Moreover, suppose that $\boldsymbol{\omega}=\mathbf{0}$ is an equilibrium state for the 
dynamic equation and that $\boldsymbol{\xi}_{0}$ is an equilibrium state for the kinematic equation. If there exists a feedback $\boldsymbol{\omega}^{*}(\boldsymbol{\xi})$ for the kinematic equation such that $\boldsymbol{\omega}^{*}(\boldsymbol{\xi}(t))$ tends to zero when $t \rightarrow \infty$ and such that $\boldsymbol{\omega}=\boldsymbol{\omega}^{*}$ renders the equilibrium in $\boldsymbol{\xi}_{0}$ asymptotically stable, then the problem of asymptotic stability of the equilibrium $\left(\mathbf{0}, \boldsymbol{\xi}_{0}\right)$ for the pair of equations is "reduced" to the search for the input $\mathbf{w}^{*}$ such that $\mathbf{F}\left(\boldsymbol{\omega}^{*}, \mathbf{w}^{*}\right)=\dot{\boldsymbol{\omega}}^{*}$. Now, the reduced problem may turn out to be rather simple when $\mathbf{F}(\cdot, \cdot)$ has a simple structure, for instance $\mathbf{F}(\boldsymbol{\omega}, \mathbf{w})=A \mathbf{w}$, where $A \in \mathbb{R}^{n \times n}$ is an invertible matrix with constant coefficients, as in a backstepping scheme. On the contrary, in other cases finding a solution may be very difficult, as in the case of the underactuated dynamics of a non-symmetric rigid body that will be analyzed in the sequel, or even impossible.

However, the successful idea on which the main result of the paper rests is to consider only piecewise constant stabilizing functions $\boldsymbol{\omega}^{*}$, what allows to explicit find the (sequence of) inputs solving the reduced problem for the underactuated rigid body dynamics.

In a second step of the analysis, the hypothesis of piecewise constant values is released: a sufficient condition is pointed out for a piecewise almost-constant (in the sense that will be specified in the sequel) $\boldsymbol{\omega}^{*}$ to globally asymptotically stabilize the zero equilibrium of the kinematic equation, while maintaining the condition that $\boldsymbol{\omega}^{*}$ tends to zero, as $\boldsymbol{\xi}$ does.

In the next section the main problem is formulated and in Section 3 a piecewise constant sequence of values of $\boldsymbol{\omega}$ is shown to globally asymptotically stabilize the zeroequilibrium of the kinematic equation. In Section 4 the generic class of systems constituted by the pair of a $d y$ namic and a kinematic equations is considered. In Section 5 some simulation results are reported showing the effectiveness of the proposed switching control law and, finally, in Section 6 some concluding remarks are drawn.

\section{Problem formulation}

This section is dedicated to present the equations describing the variation of the angular rates (dynamic equations) and of the attitude (kinematic equations) of an underactuated rigid body. The reader is referred to the available literature (see, for example, Goldstein et al. (2002); Wertz (1978)) for more details.

The acceleration of a rigid body, the motion of which in the three-dimensional space is free, can be expressed as

$$
\left\{\begin{array}{l}
J_{1} \dot{\omega}_{1}-\left(J_{2}-J_{3}\right) \omega_{2} \omega_{3}=M_{x}, \\
J_{2} \dot{\omega}_{2}-\left(J_{3}-J_{1}\right) \omega_{3} \omega_{1}=M_{y}, \\
J_{3} \dot{\omega}_{3}-\left(J_{1}-J_{2}\right) \omega_{1} \omega_{2}=M_{z},
\end{array}\right.
$$

where $\boldsymbol{\omega}=\left(\omega_{1}, \omega_{2}, \omega_{3}\right)^{\top}$ is the rotation velocity of a coordinates frame fixed to the body (w.r.t. the fixed coordinate reference $x-y-z), \mathbf{M}=\left(M_{x}, M_{y}, M_{z}\right)^{\top}$ is the torque acting on the body and $J_{1}, J_{2}$ and $J_{3}$ are the main inertia moments w.r.t. the frame fixed to the body.

Clearly, the system described by (1) is underactuated if one of the terms on the right hand side is zero; in the following, without loss of generality, the case $M_{z}=0$ is considered. Letting $u \triangleq\left(\left(J_{2}-J_{3}\right) \omega_{2} \omega_{3}+M_{x}\right) / J_{1}$, $v \triangleq\left(\left(J_{3}-J_{1}\right) \omega_{3} \omega_{1}\right) / J_{2}$ and $a=\left(J_{1}-J_{2}\right) / J_{3}$, system (1) becomes

$$
\dot{\omega}_{1}=u, \quad \dot{\omega}_{2}=v, \quad \dot{\omega}_{3}=a \omega_{1} \omega_{2},
$$

where, $u$ and $v$ are the control variables. In Equation (2) the assumption $a \neq 0$ is necessary in order to avoid the trivial equation $\dot{\omega}_{3}=0$. The condition $a \neq 0$, or equivalently $J_{x} \neq J_{y}$, implies that a non-symmetric rigid body is considered.

The equations describing the kinematics can be written in several different ways, according to the chosen parameterization (Wertz, 1978). In the following, the unit quaternion is used, namely a vector $\boldsymbol{\xi} \in \mathbb{R}^{4}$ such that $\|\boldsymbol{\xi}\|=1$, allowing a global representation of the rigid body attitude. With respect to this kind of parameterization, the equations are (e.g. Lovera and Astolfi, 2004)

$$
\dot{\boldsymbol{\xi}}=G(\boldsymbol{\omega}) \boldsymbol{\xi}
$$

where $\boldsymbol{\xi}=\left(\xi_{1}, \xi_{2}, \xi_{3}, \xi_{4}\right)^{\top}$ and

$$
G(\boldsymbol{\omega})=\frac{1}{2}\left[\begin{array}{cccc}
0 & \omega_{3} & -\omega_{2} & \omega_{1} \\
-\omega_{3} & 0 & \omega_{1} & \omega_{2} \\
\omega_{2} & -\omega_{1} & 0 & \omega_{3} \\
-\omega_{1} & -\omega_{2} & -\omega_{3} & 0
\end{array}\right]
$$

The problem addressed in the paper is the global asymptotic stabilization of the equilibrium $\boldsymbol{\xi}_{0}=(0,0,0,1)^{\top}$, $\boldsymbol{\omega}=\mathbf{0}$, of the system described by Equations (3) and (2).

\section{A stabilizing control strategy for the kine- matic equations}

In this section the dynamics of the rigid body is temporarily neglected, and the reasoning is focused only on the kinematic Equation (3). Moreover, a discontinuous time-behaviour of $\boldsymbol{\omega}$ is supposed to be allowed, what is indeed a purely ideal assumption. More specifically, for $i \in\{1,2,3\}$ the following set is defined

$$
\mathcal{O}_{i} \triangleq\left\{\boldsymbol{\omega} \text { such that } \omega_{i} \neq 0 \text { and } \omega_{j}=0 \text { for } j \neq i\right\}
$$

and the following temporary assumption is made. 
Assumption 3.1 For every pair $i, j \in\{1,2,3\}$, there exists a pair of inputs which applied to (2) cause an instantaneous variation of the vector of the angular rates from a value in $\mathcal{O}_{j}$ to a value in $\mathcal{O}_{i}$.

In the rest of the section the problem of stabilizing the equilibrium in $\boldsymbol{\xi}_{0}$ is proven to be solvable by means of a switching control law.

\subsection{Specification of the switching control scheme}

The generic structure of a switching control scheme can be summarized as follows: a set of feedback control laws is available and at each time-instant one of them is selected, to be used in the closed loop, by a decision maker according to a given switching strategy.

Thus, a switching control scheme behaves as a finite state automaton to each state of which a control law is assigned. The automaton is completely specified by defining the number of states, the sequence of the switching time-instants and the rule determining how the automaton jumps from one discrete state to the following one. Moreover, to completely specify the switching control scheme, the control law associated to each state of the automaton has to be designed. Denoting by $q$ the discrete variable associated to an automaton with $N$ states, it is natural to interpret its value as a piecewise constant function of time $q(t)$ taking values in $\{0, \ldots, N\}$.

Now, under Assumption 3.1, a switching control scheme globally asymptotically stabilizing the equilibrium in $\boldsymbol{\xi}_{0}$ of system (3) can be designed as follows: the finite state automaton associated to the scheme consists of three states $^{1}$, namely $q \in \mathcal{Q}=\{1,2,3\} ;$ moreover, chosen a positive constant $T$, the generic switching time-instant is given by

$$
\tau_{k}=k T, \quad k \in \mathbb{N} .
$$

The way in which the automaton jumps from one state to the other at the generic switching event is determined in order to maximally reduce the norm of $\left(\xi_{1}, \xi_{2}, \xi_{3}\right)^{\top}$. According to this criterion, the value of $q_{k} \triangleq \lim _{t \rightarrow \tau_{k}^{+}} q(t)$ changes as follows:

$$
q_{k}=\min \left\{i \in\{1,2,3\}:\left|\xi_{i}\left(\tau_{k}\right)\right|=\max _{j \in\{1,2,3\}}\left|\xi_{j}\left(\tau_{k}\right)\right|\right\} .
$$

Finally, as far as the control laws associated to each value of $q$ are concerned, we associate to $q=i \in\{1,2,3\}$ a

\footnotetext{
1 As it will be explained in the sequel, this choice is related to the fact that the first three coordinates of the equilibrium state are to be controlled to zero.
}

value of the velocity vector $\boldsymbol{\omega} \in \mathcal{O}_{i}$ the non-zero component of which is, for all $t \in\left[\tau_{k}, \tau_{k+1}\right)$,

$$
\bar{\omega}_{i}(\boldsymbol{\xi}(t))= \begin{cases}-\operatorname{sign}\left(\xi_{i}\left(\tau_{k}\right)\right) K \frac{\pi}{T} & \text { if } \xi_{4}\left(\tau_{k}\right)=0, \\ K \frac{2}{T} \arctan \left[-\frac{\xi_{i}\left(\tau_{k}\right)}{\left|\xi_{4}\left(\tau_{k}\right)\right|}\right] & \text { otherwise, }\end{cases}
$$

where $K$ is a constant such that $0<K<1$.

Remark 3.1 With the above choices, the angular rates are constant between two consecutive switching timeinstants, i.e. $\bar{\omega}_{i}(t) \equiv \bar{\omega}_{i}$ for all $t \in\left[\tau_{k}, \tau_{k+1}\right)$. Moreover, it is always $\operatorname{sign}\left(\bar{\omega}_{i}\right)=-\operatorname{sign}\left(\xi_{i}\left(\tau_{k}\right)\right)$ and $\frac{\bar{\omega}_{i} T}{2} \in\left(-\frac{\pi}{2}, \frac{\pi}{2}\right)$. These important facts will be exploited in the sequel. $\diamond$

In this way, the switching control scheme has been specified in all its parts; in the following subsection its stabilizing properties are shown and the choices of the quantities described so far are explained.

\subsection{Stability analysis}

For the scheme proposed in the previous subsection the following result holds.

Theorem 3.2 The switching control strategy defined by (5)-(7) globally asymptotically stabilizes the equilibrium $\boldsymbol{\xi}_{0}$ of system (3).

Proof. The thesis will be demonstrated by showing the existence of multiple Lyapunov functions (see Branicky (1998); Peleties and DeCarlo $(1991,1992))$ for the system to be controlled, what can be done by pick$\operatorname{ing}^{2} V_{i}=V[\boldsymbol{\xi}(t)]=1-\xi_{4}(t)$ for all $i \in\{1,2,3\}$. Moreover, the condition for asymptotic stability pointed out in (Liberzon, 2003, Theorem 3.1) is shown to be fulfilled; this condition ${ }^{3}$ coincides here with the existence of positive definite functions $P_{i}$ such that, for any pair of switching time-instants $\tau_{k_{1}}$ and $\tau_{k_{2}}, \tau_{k_{1}}<\tau_{k_{2}}$, such that $q\left(\tau_{k_{1}}\right)=q\left(\tau_{k_{2}}\right)=i$ and $q\left(\tau_{h}\right) \neq i$ for all $h$ such that $k_{1}<h<k_{2}$,

$$
V_{i}\left[\boldsymbol{\xi}\left(\tau_{k_{2}}\right)\right]-V_{i}\left[\boldsymbol{\xi}\left(\tau_{k_{1}}\right)\right] \leqslant-P_{i}\left[\boldsymbol{\xi}\left(\tau_{k_{1}}\right)\right] .
$$

To begin with, note that the solutions of Equation (3) can be determined for $\boldsymbol{\omega} \in \mathcal{O}_{i}$ by simple integration. In particular, for all $t \in\left[\tau_{k}, \tau_{k+1}\right)$,

$$
\xi_{4}(t)=\xi_{4}\left(\tau_{k}\right) \cos \left(\beta_{i, k}(t)\right)-\xi_{i}\left(\tau_{k}\right) \sin \left(\beta_{i, k}(t)\right),
$$

2 The chosen function is also used as Lyapunov function by Wen and Kreutz-Delgado (1991).

3 The fulfillment of condition (8) is imposed by the fact that a strictly decreasing behaviour of $V$ is not sufficient to guarantee that it tends to zero and not to a positive constant. 
where, for sake of clarity, the notation $\beta_{i, k}(t) \triangleq \bar{\omega}_{i}(t-$ $\left.\tau_{k}\right) / 2$ has been introduced. Equation (9) implies that for all $t \in\left[\tau_{k}, \tau_{k+1}\right)$,

$$
\dot{V}(t)=\frac{\bar{\omega}_{i}}{2}\left(\xi_{4}\left(\tau_{k}\right) \sin \left(\beta_{i, k}(t)\right)+\xi_{i}\left(\tau_{k}\right) \cos \left(\beta_{i, k}(t)\right)\right) .
$$

Now, notice that the case $\bar{\omega}_{i}=0$ may occur only if $\xi_{i}\left(\tau_{k}\right)=0$; this, in turn, considering how the value of $i$ is chosen, is possible if and only if $\xi_{l}\left(\tau_{k}\right)=0, \forall l \in\{1,2,3\}$. This is a trivial occurrence and the following reasoning refers to the case $\bar{\omega}_{i} \neq 0$.

Consider first the case $\xi_{4}\left(\tau_{k}\right)=0$. From the previous equation it follows that for all $t \in\left[\tau_{k}, \tau_{k+1}\right)$

$$
\left.\dot{V}(t)\right|_{\xi_{4}\left(\tau_{k}\right)=0}=\frac{\bar{\omega}_{i}}{2}\left(\xi_{i}\left(\tau_{k}\right) \cos \left(\beta_{i, k}(t)\right)\right) .
$$

Since $\frac{\bar{\omega}_{i} T}{2} \in\left(-\frac{\pi}{2}, \frac{\pi}{2}\right)$ (see Remark 3.1), the cosine in the previous expression is positive; then, as $\bar{\omega}_{i}$ and $\xi_{i}\left(\tau_{k}\right)$ have opposite sign (see again Remark 3.1), one can conclude that, for all $t \in\left[\tau_{k}, \tau_{k+1}\right),\left.\dot{V}(t)\right|_{\xi_{4}\left(\tau_{k}\right)=0} \leqslant 0$.

Now, consider the case $\xi_{4}\left(\tau_{k}\right) \neq 0$. According to the sign of $\xi_{i}\left(\tau_{k}\right)$ there can be two different situations. First suppose that $\xi_{i}\left(\tau_{k}\right) \geqslant 0$; from Remark $3.1, \bar{\omega}_{i} \leqslant 0$ and

$$
\frac{\bar{\omega}_{i} T}{2}=K \arctan \left[-\frac{\xi_{i}\left(\tau_{k}\right)}{\left|\xi_{4}\left(\tau_{k}\right)\right|}\right]>\arctan \left[-\frac{\xi_{i}\left(\tau_{k}\right)}{\left|\xi_{4}\left(\tau_{k}\right)\right|}\right] .
$$

As $\bar{\omega}_{i}<0$, one has $\frac{\bar{\omega}_{i} T}{2} \in\left(-\frac{\pi}{2}, 0\right)$ and the above relation implies that for all $t \in\left[\tau_{k}, \tau_{k+1}\right)$

$$
-\frac{\xi_{i}\left(\tau_{k}\right)}{\left|\xi_{4}\left(\tau_{k}\right)\right|}<\tan \left(\frac{\bar{\omega}_{i} T}{2}\right) \leqslant \tan \left(\beta_{i, k}(t)\right) .
$$

Moreover, as $\frac{\bar{\omega}_{i} T}{2} \in\left(-\frac{\pi}{2}, 0\right)$, for all $t \in\left[\tau_{k}, \tau_{k+1}\right)$ it is $\cos \left(\beta_{i, k}(t)\right)>0$. As a consequence, inequality (10) can be rewritten as $\xi_{i}\left(\tau_{k}\right) \cos \left(\beta_{i, k}(t)\right)+\left|\xi_{4}\left(\tau_{k}\right)\right| \sin \left(\beta_{i, k}(t)\right)>$ 0 . Finally, as $\frac{\bar{\omega}_{i} T}{2} \in\left(-\frac{\pi}{2}, 0\right)$, for all $t \in\left[\tau_{k}, \tau_{k+1}\right)$ it is $\sin \left(\beta_{i, k}(t)\right)<0$ and one obtains

$$
\begin{aligned}
\frac{2 \dot{V}(t)}{\bar{\omega}_{i}} & =\xi_{i}\left(\tau_{k}\right) \cos \left(\beta_{i, k}(t)\right)+\xi_{4}\left(\tau_{k}\right) \sin \left(\beta_{i, k}(t)\right) \geqslant \\
& \geqslant \xi_{i}\left(\tau_{k}\right) \cos \left(\beta_{i, k}(t)\right)+\left|\xi_{4}\left(\tau_{k}\right)\right| \sin \left(\beta_{i, k}(t)\right)>0
\end{aligned}
$$

for all $t \in\left[\tau_{k}, \tau_{k+1}\right)$ and, in conclusion, $\dot{V}(t)<0$ for all $t \in\left[\tau_{k}, \tau_{k+1}\right)$. A similar reasoning can be done in the case $\xi_{i}\left(\tau_{k}\right)<0$.

The proof can be concluded by noticing that the function $P_{i}(\boldsymbol{\xi}) \triangleq \xi_{i} \sin \left(\bar{\omega}_{i} T / 2\right)+\left|\xi_{4}\right|\left(1-\cos \left(\bar{\omega}_{i} T / 2\right)\right)$ is such that condition (8) is fulfilled.

Thus the switching control scheme globally asymptotically stabilizes the equilibrium in $\boldsymbol{\xi}_{0}$ of (3).

\section{A stabilizing control strategy for the whole system}

Now the dynamic equation, neglected so far, is considered and the idea presented in the previous section is exploited in the case of the complete system described by

$$
\left\{\begin{array}{l}
\dot{\boldsymbol{\omega}}=F_{1}(\boldsymbol{\omega})+F_{2}(\boldsymbol{\omega}) \mathbf{w} \\
\dot{\boldsymbol{\xi}}=G(\boldsymbol{\omega}) \boldsymbol{\xi}
\end{array}\right.
$$

where $F_{1}(\boldsymbol{\omega})=\left(0,0, a \omega_{1} \omega_{2}\right)^{\top}, F_{2}(\boldsymbol{\omega})=\left(\begin{array}{lll}1 & 0 & 0 \\ 0 & 1 & 0\end{array}\right)^{\top}, \mathbf{w}=$ $(u, v)^{\top}$ and $G(\boldsymbol{\omega})$ is defined as in (4).

In this case the angular velocities must have a continuous time-behavior, Assumption 3.1 does not hold and the switching control law presented in the previous section cannot be used. Nevertheless, an approximated form of it (that will be specified in the following) can be shown to provide global asymptotic stability of the equilibrium $\left(\mathbf{0}, \boldsymbol{\xi}_{0}\right)$ of system (11). This is done in the following by using the fact that, in order to prove stability of the desired equilibrium of a hybrid system, a candidate Lyapunov function needs to have a negative definite time-derivative along the trajectory only in some time-intervals, and not for all time, provided that some boundedness conditions are fulfilled. The idea is not new (see, e.g. (Brogliato et al., 1997)); however, it is exploited here in a different way leading to a novel stability theorem.

The following definition is first introduced.

Definition 4.1 A continuous function $F: \mathbb{R}^{+} \rightarrow \mathbb{R}^{+}$is almost non-increasing (ANI) if there exists a sequence of intervals $\left\{\left[a_{i}, b_{i}\right]\right\}_{i \in \mathbb{N}}$, with $a_{i} \in[0,+\infty), b_{i} \in[0,+\infty)$ and $a_{i}<b_{i}<a_{i+1}$ such that

(i) $F$ is non-increasing over $\left(b_{i}, a_{i+1}\right)$ for all $i$ and over $\left(0, a_{1}\right)$ if $a_{1} \neq 0$

(ii) there exists a positive constant $L$ such that, for all $i$ and all $x \in\left[a_{i}, b_{i}\right], F(x) \leqslant L F\left(a_{i}\right)$;

(iii) the sequence $\left\{F\left(a_{i}\right)\right\}_{i \in \mathbb{N}}$ is non-increasing: for all $i$ one has $F\left(a_{i+1}\right) \leqslant F\left(a_{i}\right)$.

In Figure 1 an example of an ANI function is reported.

Note that the ANI behaviour implies, in particular, that for all $t_{0} \in \mathbb{R}+$,

$$
F(t) \leqslant L F\left(t_{0}\right), \quad \forall t \geqslant t_{0} .
$$

Definition 4.1 is helpful to prove the following theorem.

Theorem 4.1 Consider an autonomous dynamic system $\Sigma: \dot{\mathbf{x}}=\mathbf{f}(\mathbf{x})$, where $\mathbf{x}$ belongs to some manifold $M \subset \mathbb{R}^{n}$, such that $\mathbf{0} \in M$, and $\mathbf{f}: M \rightarrow T M$ is continuous and such that the origin is an equilibrium state: 


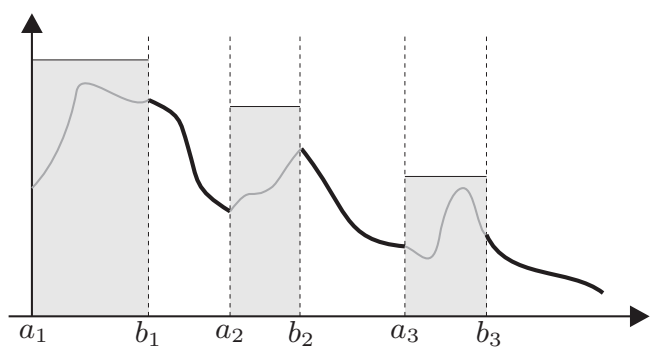

Fig. 1. Example of an ANI function. The behaviour of the function in the intervals $\left[a_{i}, b_{i}\right]$ does not affect the overall decreasing behaviour, provided that the function does not grow beyond the upper edge of the shaded rectangles. In the particular case depicted in the figure $L=2$.

$\mathbf{f}(\mathbf{0})=\mathbf{0}$. If there exists a continuous positive definite and proper function ${ }^{4} V: M \rightarrow \mathbb{R}^{+}$such that $V(\mathbf{x}(t))$ is ANI (as a function of time), then the zero equilibrium of $\Sigma$ is stable in the sense of Lyapunov. Moreover, if there exists a positive definite function $\gamma: \mathbb{R}^{n} \rightarrow \mathbb{R}$ such that

$$
a_{i}<a_{j} \quad \Rightarrow V\left[\mathbf{x}\left(a_{j}\right)\right]-V\left[\mathbf{x}\left(a_{i}\right)\right] \leqslant-\gamma\left(\mathbf{x}\left(a_{i}\right)\right)
$$

then the zero equilibrium of $\Sigma$ is globally asymptotically stable.

Proof. In order to prove Lyapunov stability, one needs to show that, for every neighborhood $\mathcal{U}_{\mathbf{0}}$ of the origin, there exists a neighborhood $\mathcal{V}_{\mathbf{0}}$ (of the origin) such that if $\mathbf{x}(0) \in \mathcal{V}_{\mathbf{0}}$ then $\mathbf{x}(t) \in \mathcal{U}_{\mathbf{0}}$ for all $t>0$.In fact, choose $R$ such that $\|\mathbf{x}\| \leqslant R \Rightarrow \mathbf{x} \in \mathcal{U}_{\mathbf{0}}$ and let $V_{1} \triangleq \min _{\|\mathbf{y}\|=R} V(\mathbf{y})$. By the continuity of $V$, it is always possible to choose a value $r$ such that if $\|\mathbf{x}\|<r$ then $V(\mathbf{x})<V_{1} / L$. Then $\mathcal{V}_{\mathbf{0}}=\{\mathbf{x}:\|\mathbf{x}\|<r\}$. In fact, pick $\mathbf{x}(0) \in \mathcal{V}_{\mathbf{0}}$ and suppose, ab absurdo, that the trajectory exits $\mathcal{U}_{\mathbf{0}}$; this would mean that it first intersects the sphere $\mathcal{S} \triangleq\{\mathbf{x}:\|\mathbf{x}\|=R\}$ which is included in $\mathcal{U}_{\mathbf{0}}$. As a consequence, there should exist a time $\bar{t}$ such that $\mathbf{x}(\bar{t}) \in \mathcal{S}$ which, in turn, would mean $V[\mathbf{x}(\bar{t})] \geqslant V_{1}$. This, as $V[\mathbf{x}(0)]<V_{1} / L$, is in contradiction with Equation (12).

To prove global asymptotic stability, note that condition (13) implies in particular that the sequence $\left\{V\left(\mathbf{x}\left(a_{i}\right)\right)\right\}_{i \in \mathbb{N}}$ is strictly decreasing, hence it admits a limit $V_{\infty} \triangleq \lim _{i \rightarrow \infty} V\left[\mathbf{x}\left(a_{i}\right)\right]$, i.e. for all $\varepsilon>0$ there exists $j$ such that, for all $i>j,\left|V\left[\mathbf{x}\left(a_{i}\right)\right]-V_{\infty}\right|<\varepsilon$. This implies that for all $\varepsilon>0$ there exists $j$ such that for all $i_{1}, i_{2}>j$ $\left|V\left[\mathbf{x}\left(a_{i_{1}}\right)\right]-V\left[\mathbf{x}\left(a_{i_{2}}\right)\right]\right|<\varepsilon$ and, in turn,

$$
\lim _{i \rightarrow \infty}\left|V\left[\mathbf{x}\left(a_{i}\right)\right]-V\left[\mathbf{x}\left(a_{i+1}\right)\right]\right|=0 .
$$

Suppose now that $V_{\infty} \neq 0$; as $V(\mathbf{x}) \neq 0$ for $\mathbf{x} \neq 0$, this

$\overline{4}$ A continuous function $V: M \rightarrow \mathbb{R}$, with $M \subset \mathbb{R}^{n}$, is said to be positive definite if $V(\mathbf{0})=0$ and $V(\mathbf{x})>0$ for all $\mathbf{x} \neq 0$, and proper if, for any $a \in \mathbb{R}$, the set $V^{-1}([0, a])=\left\{\mathbf{x} \in \mathbb{R}^{n}\right.$ : $0 \leqslant V(x) \leqslant a\}$ is compact (see (Isidori, 1995, Appendix B)). implies that $\lim _{i \rightarrow \infty} \mathbf{x}\left(a_{i}\right)$ either does not exist or is different from zero. Hence, by hypothesis, for every $j$ it is always possible to find $k>j$ such that $\gamma\left(\mathbf{x}\left(a_{k}\right)\right)>\varepsilon$ for some $\varepsilon>0$. This, together with (13), implies $V\left[\mathbf{x}\left(a_{k}\right)\right]-$ $V\left[\mathbf{x}\left(a_{k+1}\right)\right] \geqslant \gamma\left(a_{k}\right)>\varepsilon$, which is in contradiction with (14). Then it must be $\lim _{i \rightarrow \infty} V\left[\mathbf{x}\left(a_{i}\right)\right]=0$, i.e. for all $\varepsilon>0$ there exists $j$ such that, for all $i>j, V\left[\mathbf{x}\left(a_{i}\right)\right]<\varepsilon$, what implies $\lim _{t \rightarrow \infty} V[\mathbf{x}(t)]=0$ and finally $\lim _{t \rightarrow \infty} \mathbf{x}(t)=\mathbf{0}$.

In addition, the following lemma is given the proof of which is straightforward and therefore omitted.

Lemma 4.2 Consider system (2). Given a positive constant $h$, for each pair of vectors $\boldsymbol{\omega}_{P}=\left(\omega_{1 P}, \omega_{2 P}, \omega_{3 P}\right)^{\top}$ and $\boldsymbol{\omega}_{D}=\left(\omega_{1 D}, \omega_{2 D}, \omega_{3 D}\right)^{\top}$, there exist two constants $u_{0}=u_{0}\left(\boldsymbol{\omega}_{P}, \boldsymbol{\omega}_{D}\right)$ and $v_{0}=v_{0}\left(\boldsymbol{\omega}_{P}, \boldsymbol{\omega}_{D}\right)$ such that, if $\boldsymbol{\omega}(t)=\boldsymbol{\omega}_{P}$ and

$$
\mathbf{w}(t)= \begin{cases}{\left[\frac{\omega_{1 D}-\omega_{1 P}}{h}, \frac{\omega_{2 D}-\omega_{2 P}}{h}\right]^{\top}} & \text { for } t \in \mathcal{I}_{1}, \\ {\left[u_{0}, 0\right]^{\top}} & \text { for } t \in \mathcal{I}_{2}, \\ {\left[0, v_{0}\right]^{\top}} & \text { for } t \in \mathcal{I}_{3}, \\ {\left[-u_{0}, 0\right]^{\top}} & \text { for } t \in \mathcal{I}_{4}, \\ {\left[0,-v_{0}\right]^{\top}} & \text { for } t \in \mathcal{I}_{5},\end{cases}
$$

where $\mathcal{I}_{m}$ is the (left-open and right-closed) interval

$$
\mathcal{I}_{m} \triangleq(t+(m-1) h, t+m h], \quad m=1, \ldots, 5,
$$

then $\boldsymbol{\omega}(t+5 h)=\boldsymbol{\omega}_{D}$.

With the help of Lemma 4.2 it is possible to design a sequence of controls to drive the vector of the angular rates from $\boldsymbol{\omega}\left(\tau_{k}\right)=\boldsymbol{\omega}_{P} \in \mathcal{O}_{i}$ to $\boldsymbol{\omega}\left(\tau_{k}+5 h_{k}\right)=\boldsymbol{\omega}_{D} \in \mathcal{O}_{j}$ in a finite time $5 h_{k}$ (see Figure 2, where an example is depicted). One of the main goals of the remainder of the section is to show how the value of $h_{k}$ can be properly select in order to ensure global asymptotic stability.

\subsection{The switching control strategy}

In this subsection the switching control scheme for the whole system is described. Subsequently, its stabilizing properties are analyzed. To begin with, suppose that a positive constant $h_{k}$ (the value of which will be specified in the sequel) is assigned to each switching time-instant $\tau_{k}$. Let $\widetilde{\tau}_{k} \triangleq \tau_{k}+5 h_{k}$ and

$$
T_{S}\left(\tau_{k}\right) \triangleq \min \left\{t>\widetilde{\tau}_{k}: \dot{\xi}_{4}(t)=0\right\},
$$

and, in respect to these quantities, consider the following modified switching control strategy. 


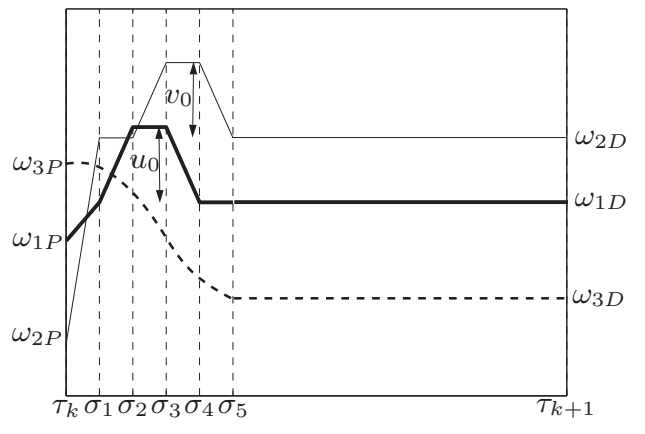

Fig. 2. Example of the time histories of the angular rates between one switching time instant $\left(\tau_{k}\right)$ and the following $\left(\tau_{k+1}\right)$. The notation $\sigma_{j}$, for $j \in\{1,2,3,4,5\}$, has been used instead of $\tau_{k}+j h_{k}$.

S1) The switching time-instants are defined by: $\tau_{k+1}=$ $T_{S}\left(\tau_{k}\right)$.

S2) The value of $q$ is updated according to (6).

S3) For $t \in\left[\tau_{k}, \widetilde{\tau}_{k}\right)$ inputs are selected as specified by (15), with $\boldsymbol{\omega}_{P}=\lim _{t \rightarrow \tau_{k}^{-}} \boldsymbol{\omega}(t), \omega_{i D}=\bar{\omega}_{i}$ for $i=q_{k}$ and $\omega_{i D}=0$ for $i \neq q_{k}$. For $t \in\left[\widetilde{\tau}_{k}, \tau_{k+1}\right)$ inputs are set to zero. The value of $u_{0}$ and $v_{0}$ can be determined analytically as in the following example.

Example 4.1 Consider the transition from $\boldsymbol{\omega}_{\boldsymbol{P}}=$ $\left(0,0, \omega_{3 P}\right)^{\top} \in \mathcal{O}_{3}$ to $\boldsymbol{\omega}_{D}=\left(\omega_{1 D}, 0,0\right)^{\top} \in \mathcal{O}_{1}$. From Equation (2) it is easy to compute the variation of the three angular rates for the five intervals $\mathcal{I}_{m}$.

- For $t \in \mathcal{I}_{1}: \omega_{1}(t)=\omega_{1 D}\left(t-\tau_{k}\right) / h_{k}, \omega_{2}(t)=0, \omega_{3}(t)=$ $\omega_{3 P}$.

- For $t \in \mathcal{I}_{2}: \omega_{1}(t)=\omega_{1 D}+u_{0}\left(t-\tau_{k}-h_{k}\right), \omega_{2}(t)=0$, $\omega_{3}(t)=\omega_{3 P}$

- For $t \in \mathcal{I}_{3}: \omega_{1}(t)=\omega_{1 D}+u_{0} h_{k}, \omega_{2}(t)=v_{0}\left(t-\tau_{k}-2 h_{k}\right)$, $\omega_{3}(t)=\omega_{3 P}+a\left(h_{k} u_{0}+\omega_{1 D}\right) v_{0}\left(t-\tau_{k}-2 h_{k}\right)^{2} / 2$.

- For $t \in \mathcal{I}_{4}: \omega_{1}(t)=\omega_{1 D}+u_{0}\left(\tau_{k}+4 h_{k}-t\right), \omega_{2}(t)=v_{0} h_{k}$, $\omega_{3}(t)=\omega_{3 P}+a\left(h_{k} u_{0}+\omega_{1 D}\right) v_{0} h_{k}^{2} / 2+a h_{k} v_{0} \omega_{1 D}\left(t-\tau_{k}-\right.$ $\left.3 h_{k}\right)+a h_{k}^{2} u_{0} v_{0}\left(t-\tau_{k}-3 h_{k}\right)-a u_{0} v_{0} h_{k}\left(t-\tau_{k}-3 h_{k}\right)^{2} / 2$.

- For $t \in \mathcal{I}_{5}: \omega_{1}(t)=\omega_{1 D}, \omega_{2}(t)=v_{0} h_{k}-v_{0}\left(t-\tau_{k}-4 h_{k}\right)$, $\omega_{3}(t)=\omega_{3 P}+a \frac{3}{2} v_{0} \omega_{1 D} h_{k}^{2}+a u_{0} v_{0} h_{k}^{3}+a \omega_{1 D} v_{0} h_{k}\left(t-\tau_{k}-\right.$ $\left.4 h_{k}\right)-a \omega_{1 D} v_{0}\left(t-\tau_{k}-4 h_{k}\right)^{2} / 2$

Therefore $u_{0}$ and $v_{0}$ have to be selected in such a way that $\omega_{3}\left(\tau_{k}+5 h_{k}\right)=\omega_{3 P}+2 a v_{0} \omega_{1 D} h_{k}^{2}+a u_{0} v_{0} h_{k}^{3}=0$. For instance, $u_{0}=\omega_{1 D}\left(\sqrt{1+\left|\omega_{3 P}\right| /\left(a h_{k} \omega_{1 D}^{2}\right)}-1\right) / h_{k}$ and $v_{0}=-\operatorname{sign}\left(\omega_{3 P}\right) u_{0}$.

Remark 4.1 The switching control scheme described above is a "sample-and-hold" feedback control. In fact, in the interval between two consecutive switching timeinstants, i.e. for $t \in\left[\tau_{k}, \tau_{k+1}\right)$, the value of the control signals do not depend on $\boldsymbol{\xi}(t)$, namely on the actual value of the state at time $t$, but on $\boldsymbol{\xi}\left(\tau_{k}\right)$, namely on the value of the state at the beginning of the interval.

\subsection{Stability analysis}

This subsection is dedicated to show that $h_{k}$ can be chosen in such a way that the hypotheses of Theorem 4.1 are fulfilled thus proving the global asymptotic stabilizability of system (11). In particular, the function $V(\boldsymbol{\xi}(t))=$ $1-\xi_{4}(t)$ is proven to be almost non-increasing and condition (13) is shown to be fulfilled. To this end, first some useful quantities are introduced:

$$
\begin{aligned}
& \omega_{M}\left(\tau_{k}\right) \triangleq \max _{j \in\{1,2,3\}}\left[\max _{t \in\left[\tau_{k}, \tilde{\tau}_{k}\right]}\left|\omega_{j}(t)\right|\right], \\
& \xi_{M}\left(\tau_{k}\right) \triangleq \max _{j \in\{1,2,3\}}\left[\max _{t \in\left[\tau_{k}, \tilde{\tau}_{k}\right]}\left|\xi_{j}(t)\right|\right], \\
& l(i, \boldsymbol{\xi}) \triangleq \frac{1}{3}\left(-3\left|\xi_{4}\right|-1+\sqrt{9 \xi_{4}^{2}+1+6\left|\xi_{4}\right|+3 \xi_{i}^{2}}\right), \\
& H(i, \boldsymbol{\xi}) \triangleq \min \left\{\left|\xi_{4}\right|,\left|\xi_{i}\right|, l(i, \boldsymbol{\xi})\right\} .
\end{aligned}
$$

Moreover, note that the following lemmas hold (the proofs of Lemmas 4.3 and 4.4 can be found in the Appendix while the proof of Lemma 4.5 is trivial and left to the reader).

Lemma 4.3 Consider Equation (3). For each $i \in$ $\{1,2,3\}$, for each $\xi_{i}\left(\tau_{k}\right) \neq 0$ and for each $h_{k}>0$, if $\omega_{M}\left(\tau_{k}\right)$ is defined as in Equation (17) and if $\frac{1}{5} \omega_{M}\left(\tau_{k}\right) h_{k}<2\left|\xi_{i}\left(\tau_{k}\right)\right|$ then $\operatorname{sign}\left[\xi_{i}\left(\widetilde{\tau}_{k}\right)\right]=\operatorname{sign}\left[\xi_{i}\left(\tau_{k}\right)\right]$ and, in particular, $\xi_{i}\left(\widetilde{\tau}_{k}\right) \neq 0$.

Lemma 4.4 Consider Equation (3). For each $i \in$ $\{1,2,3\}$ and for each $\xi_{i}\left(\tau_{k}\right) \neq 0$, if $H(i, \boldsymbol{\xi})$ is defined as in Equation (20) and $h_{k}$ is such that $15 \omega_{M}\left(\tau_{k}\right) h_{k} \leqslant$ $H\left(i, \boldsymbol{\xi}\left(\tau_{k}\right)\right)$, then defining $\rho\left(\widetilde{\tau}_{k}\right) \triangleq \xi_{4}^{2}\left(\widetilde{\tau}_{k}\right)+\xi_{i}^{2}\left(\widetilde{\tau}_{k}\right)$, $\sqrt{\rho\left(\widetilde{\tau}_{k}\right)}-\xi_{4}\left(\widetilde{\tau}_{k}\right)>15 \omega_{M}\left(\tau_{k}\right) \xi_{M}\left(\tau_{k}\right) h_{k}$.

Lemma 4.5 Let $f(t)=a \sin (\Omega t)+b \cos (\Omega t)$, with $a, b$ and $\Omega \in \mathbb{R} \backslash\{0\}$, and $\bar{t} \triangleq \min \{t>0: f(t)=0\}$. Then $\cos (\Omega \bar{t})=-\operatorname{sign}(a b \Omega) \sqrt{a^{2} /\left(a^{2}+b^{2}\right)}$.

Now the main result of the paper can be proven.

Theorem 4.6 Let a switching control scheme with discrete state $q \in\{1,2,3\}$ be applied to the system defined by Equation (3). If the switching control strategy is defined by $S 1, S 2$ and $S 3$ with

$$
h_{k}=\min \left\{\frac{H\left(i, \boldsymbol{\xi}\left(\tau_{k}\right)\right)}{15 \omega_{M}\left(\tau_{k}\right)}, \frac{1-\left|\xi_{4}\left(\tau_{k}\right)\right|}{15 \omega_{M}\left(\tau_{k}\right)}\right\}
$$

then, the sequence of intervals $\left\{\tau_{k}, \widetilde{\tau}_{k}\right\}_{k \in \mathbb{N}}$ is such that the function $V(t)=V[\boldsymbol{\xi}(t)]=1-\xi_{4}(t)$ is ANI. Moreover, there exists a positive definite function $\gamma: \mathbb{R}^{4} \rightarrow \mathbb{R}$ such that, for all $k \in \mathbb{N}, V\left[\boldsymbol{\xi}\left(\tau_{k+1}\right)\right]-V\left[\boldsymbol{\xi}\left(\tau_{k}\right)\right] \leqslant-\gamma\left(\boldsymbol{\xi}\left(\tau_{k}\right)\right)$. 
Proof. To begin with, note that in the interval $\left[\widetilde{\tau}_{k}, \tau_{k+1}\right)$, supposing $q_{k}=i$, the vector of angular rates has a constant value $\boldsymbol{\omega}(t)=\overline{\boldsymbol{\omega}} \in \mathcal{O}_{i}$ and the expression of $\xi_{4}(t)$ can be easily computed by integration of Equation (3)

$$
\xi_{4}(t)=\xi_{4}\left(\widetilde{\tau}_{k}\right) \cos \left(\widetilde{\beta}_{i, k}(t)\right)-\xi_{i}\left(\widetilde{\tau}_{k}\right) \sin \left(\widetilde{\beta}_{i, k}(t)\right),
$$

where $\widetilde{\beta}_{i, k}(t) \triangleq \bar{\omega}_{i}\left(t-\widetilde{\tau}_{k}\right) / 2$. As a consequence,

$$
\dot{\xi}_{4}(t)=-\frac{\bar{\omega}_{i}}{2}\left(\xi_{4}\left(\widetilde{\tau}_{k}\right) \sin \left(\widetilde{\beta}_{i, k}(t)\right)+\xi_{i}\left(\widetilde{\tau}_{k}\right) \cos \left(\widetilde{\beta}_{i, k}(t)\right)\right) .
$$

Observe, now, that the chosen value of $h_{k}$ is such that $15 \omega_{M}\left(\tau_{k}\right) h_{k} \leqslant\left|\xi_{i}\left(\tau_{k}\right)\right|<2\left|\xi_{i}\left(\tau_{k}\right)\right|$, hence Lemma 4.3 holds, i.e. $\operatorname{sign}\left[\xi_{i}\left(\widetilde{\tau}_{k}\right)\right]=\operatorname{sign}\left[\xi_{i}\left(\tau_{k}\right)\right]$. This, recalling Remark 3.1 and Equation (22), implies that $\dot{\xi}_{4}\left(\widetilde{\tau}_{k}\right)=-\bar{\omega}_{i} \xi_{i}\left(\widetilde{\tau}_{k}\right) / 2>0$ which in turn, considering how $\tau_{k+1}$ is chosen (Equations (16) and S1), yields $\dot{\xi}_{4}(t)>0$ for all $t \in\left(\widetilde{\tau}_{k}, \tau_{k+1}\right)$ and, in conclusion, $\dot{V}(t)<0$ for all $t \in\left(\widetilde{\tau}_{k}, \tau_{k+1}\right)$ and (i) in Definition 4.1 holds.

Now, from Equation (3) it is easy to compute $\dot{\xi}_{4}(t)=$ $-\frac{1}{2} \sum_{i=1}^{3} \omega_{i}(t) \xi_{i}(t)$ which implies (see (17) and (18)) that for all $t \in\left[\tau_{k}, \widetilde{\tau}_{k}\right]$,

$$
\left|\dot{\xi}_{4}(t)\right| \leqslant \frac{1}{2} \omega_{M}\left(\tau_{k}\right) \sum_{i=1}^{3}\left|\xi_{i}(t)\right| \leqslant \frac{3}{2} \omega_{M}\left(\tau_{k}\right) \xi_{M}\left(\tau_{k}\right)
$$

Hence,

$$
\begin{aligned}
& \left|\xi_{4}\left(\tau_{k}\right)-\xi_{4}(t)\right| \leqslant \frac{3}{2} \omega_{M}\left(\tau_{k}\right) \xi_{M}\left(\tau_{k}\right)\left(t-\tau_{k}\right) \leqslant \\
& \leqslant \frac{15}{2} \omega_{M}\left(\tau_{k}\right) \xi_{M}\left(\tau_{k}\right) h_{k}, \quad \forall t \in\left[\tau_{k}, \widetilde{\tau}_{k}\right] .
\end{aligned}
$$

Now, if $\xi_{4}(t) \geqslant \xi_{4}\left(\tau_{k}\right)$ for all $t \in\left[\tau_{k}, \widetilde{\tau}_{k}\right]$, then $V(t)=1-$ $\xi_{4}(t) \leqslant 1-\xi_{4}\left(\tau_{k}\right)=V\left(\tau_{k}\right)$ and (ii) in Definition 4.1 holds with $L=1$. On the other hand, if $\xi_{4}(t)<\xi_{4}\left(\tau_{k}\right)$ for some $t \in\left[\tau_{k}, \widetilde{\tau}_{k}\right]$, Equation (23) becomes, by the definition of $h_{k}$,

$$
\begin{aligned}
\xi_{4}\left(\tau_{k}\right)-\xi_{4}(t) & \leqslant \frac{\xi_{M}\left(\tau_{k}\right)}{2}\left(1-\left|\xi_{4}\left(\tau_{k}\right)\right|\right) \leqslant \\
\leqslant & \frac{1}{2}\left(1-\xi_{4}\left(\tau_{k}\right)\right), \quad \forall t \in\left[\tau_{k}, \widetilde{\tau}_{k}\right],
\end{aligned}
$$

where the last inequality holds as $\xi_{M}(t) \leqslant 1$. Equation (24) implies that for all $t \in\left[\tau_{k}, \widetilde{\tau}_{k}\right], V(t)=1-\xi_{4}(t) \leqslant$ $3\left(1-\xi_{4}\left(\tau_{k}\right)\right) / 2=3 V\left(\tau_{k}\right) / 2$ and (ii) holds with $L=3 / 2$.
Divide the interval $\left[\tau_{k}, \tau_{k+1}\right)$ into two subintervals, $\left[\tau_{k}, \widetilde{\tau}_{k}\right)$ and $\left[\widetilde{\tau}_{k}, \tau_{k+1}\right)$. From Equation $(21)$

$$
\begin{aligned}
\xi_{4}\left(\tau_{k+1}\right)=\xi_{4}\left(\widetilde{\tau}_{k}\right) \cos & \left(\frac{\bar{\omega}_{i}}{2} T_{S}^{\prime}\left(\tau_{k}\right)\right)- \\
& -\xi_{i}\left(\widetilde{\tau}_{k}\right) \sin \left(\frac{\bar{\omega}_{i}}{2} T_{S}^{\prime}\left(\tau_{k}\right)\right)
\end{aligned}
$$

where $T_{S}^{\prime}\left(\tau_{k}\right)=T_{S}\left(\tau_{k}\right)-\widetilde{\tau}_{k}$. Moreover, the definition of $T_{S}\left(\tau_{k}\right)$ yields $\dot{\xi}_{4}\left(\tau_{k+1}\right)=0$ and, as a consequence, from Equation (22) one obtains:

$$
\xi_{4}\left(\widetilde{\tau}_{k}\right) \sin \left(\frac{\bar{\omega}_{i}}{2} T_{S}^{\prime}\left(\tau_{k}\right)\right)+\xi_{i}\left(\widetilde{\tau}_{k}\right) \cos \left(\frac{\bar{\omega}_{i}}{2} T_{S}^{\prime}\left(\tau_{k}\right)\right)=0
$$

In Equation (26) the case $\bar{\omega}_{i}=0$ is excluded as it would mean, by the definition of $\bar{\omega}_{i}, \xi_{i}=0$ for all $i \in\{1,2,3\}$. Now, according to the value of $\xi_{4}\left(\widetilde{\tau}_{k}\right)$, two cases have to be considered. Consider first the case $\xi_{4}\left(\widetilde{\tau}_{k}\right) \neq 0$. Equation (26) can be rewritten as:

$$
\sin \left[\frac{\bar{\omega}_{i}}{2} T_{S}^{\prime}\left(\tau_{k}\right)\right]=-\frac{\xi_{i}\left(\widetilde{\tau}_{k}\right)}{\xi_{4}\left(\widetilde{\tau}_{k}\right)} \cos \left[\frac{\bar{\omega}_{i}}{2} T_{S}^{\prime}\left(\tau_{k}\right)\right] .
$$

Substituting (27) into (25) yields

$$
\xi_{4}\left(\tau_{k+1}\right)=\left[\xi_{4}\left(\widetilde{\tau}_{k}\right)+\frac{\xi_{i}^{2}\left(\widetilde{\tau}_{k}\right)}{\xi_{4}\left(\widetilde{\tau}_{k}\right)}\right] \cos \left[\frac{\bar{\omega}_{i}}{2} T_{S}^{\prime}\left(\tau_{k}\right)\right] .
$$

Moreover, Lemma 4.5 with $a=\xi_{4}\left(\widetilde{\tau}_{k}\right), b=\xi_{i}\left(\widetilde{\tau}_{k}\right)$ and $\Omega=\bar{\omega}_{i} / 2$, yields

$$
\cos \left[\frac{\bar{\omega}_{i}}{2} T_{S}^{\prime}\left(\tau_{k}\right)\right]=-\operatorname{sign}\left[\xi_{i}\left(\widetilde{\tau}_{k}\right) \xi_{4}\left(\widetilde{\tau}_{k}\right) \bar{\omega}_{i}\right] \sqrt{\frac{\xi_{4}^{2}\left(\widetilde{\tau}_{k}\right)}{\rho\left(\widetilde{\tau}_{k}\right)}}
$$

where $\rho\left(\widetilde{\tau}_{k}\right)=\xi_{i}^{2}\left(\widetilde{\tau}_{k}\right)+\xi_{4}^{2}\left(\widetilde{\tau}_{k}\right)$. Substituting (29) into (28) yields $\xi_{4}\left(\tau_{k+1}\right)=-\operatorname{sign}\left[\xi_{i}\left(\widetilde{\tau}_{k}\right) \bar{\omega}_{i}\right] \sqrt{\rho\left(\widetilde{\tau}_{k}\right)}$. By Lemma 4.3 and Remark 3.1, one obtains $\xi_{4}\left(\tau_{k+1}\right)=\sqrt{\rho\left(\widetilde{\tau}_{k}\right)}$. Finally, note that, with the chosen value of $h_{k}$, Lemma 4.4 holds and yields

$$
\begin{aligned}
\xi_{4}\left(\tau_{k+1}\right)-\xi_{4}\left(\widetilde{\tau}_{k}\right)=\sqrt{\rho\left(\widetilde{\tau}_{k}\right)}-\xi_{4}\left(\widetilde{\tau}_{k}\right)> \\
15 \omega_{M}\left(\tau_{k}\right) \xi_{M}\left(\tau_{k}\right) h_{k}
\end{aligned}
$$

Moreover, by using Equation (23) with $t=\widetilde{\tau}_{k}$,

$$
\begin{aligned}
-\xi_{4}\left(\widetilde{\tau}_{k}\right)+\xi_{4}\left(\tau_{k}\right) \leqslant \mid & -\xi_{4}\left(\widetilde{\tau}_{k}\right)+\xi_{4}\left(\tau_{k}\right) \mid \leqslant \\
& \leqslant \frac{15}{2} \omega_{M}\left(\tau_{k}\right) \xi_{M}\left(\tau_{k}\right) h_{k} .
\end{aligned}
$$

Putting together Equations (30) and (31) one obtains $\xi_{4}\left(\tau_{k+1}\right)-\xi_{4}\left(\widetilde{\tau}_{k}\right)>-\xi_{4}\left(\widetilde{\tau}_{k}\right)+\xi_{4}\left(\tau_{k}\right)$, from which $V\left(\tau_{k+1}\right)<V\left(\tau_{k}\right)$ immediately follows.

In the case $\xi_{4}\left(\widetilde{\tau}_{k}\right)=0$, from Equation (26) and considering that from Lemma $4.3 \xi_{i}\left(\widetilde{\tau}_{k}\right) \neq 0$, it follows that 
$\cos \left(\bar{\omega}_{i} T_{S}^{\prime}\left(\tau_{k}\right) / 2\right)=0$ and so from Remark 3.1, Lemma 4.3 and $(25)$ one obtains $\xi_{4}\left(\tau_{k+1}\right)=\left|\xi_{i}\left(\widetilde{\tau}_{k}\right)\right|$. Lemma 4.4 in this case yields $\left|\xi_{i}\left(\widetilde{\tau}_{k}\right)\right|>15 \omega_{M}\left(\tau_{k}\right) \xi_{M}\left(\tau_{k}\right) h_{k}$, while (31) reads $\xi_{4}\left(\tau_{k}\right) \leqslant 15 \omega_{M}\left(\tau_{k}\right) \xi_{M}\left(\tau_{k}\right) h_{k} / 2$; then also in this case $V\left(\tau_{k+1}\right)<V\left(\tau_{k}\right)$. In conclusion, $V(t)$ is ANI.

To prove the second part of the statement, notice that the following relations hold:

$$
\begin{gathered}
\xi_{4}\left(\tau_{k+1}\right)-\xi_{4}\left(\widetilde{\tau}_{k}\right)>15 \omega_{M}\left(\tau_{k}\right) \xi_{M}\left(\tau_{k}\right) h_{k}= \\
\frac{15}{2} \omega_{M}\left(\tau_{k}\right) \xi_{M}\left(\tau_{k}\right) h_{k}+\frac{15}{2} \omega_{M}\left(\tau_{k}\right) \xi_{M}\left(\tau_{k}\right) h_{k} \geqslant \\
\geqslant-\xi_{4}\left(\widetilde{\tau}_{k}\right)+\xi_{4}\left(\tau_{k}\right)+\frac{15}{2} \omega_{M}\left(\tau_{k}\right) \xi_{M}\left(\tau_{k}\right) h_{k},
\end{gathered}
$$

which yield $\xi_{4}\left(\tau_{k+1}\right)-\xi_{4}\left(\tau_{k}\right) \geqslant 15 \omega_{M}\left(\tau_{k}\right) \xi_{M}\left(\tau_{k}\right) h_{k} / 2$. Now, defining the quantities:

$$
\hat{i}(\boldsymbol{\xi}) \triangleq \min \left\{l \in\{1,2,3\}:\left|\xi_{l}\right|=\max _{i \in\{1,2,3\}}\left|\xi_{i}\right|\right\},
$$

and $\widehat{H}(\boldsymbol{\xi}) \triangleq \min \left\{\left|\xi_{\hat{i}}\right|, l(\hat{i}, \boldsymbol{\xi}), 1-\left|\xi_{4}\right|,\left|\xi_{4}\right|\right\}$, it is easy to see that $15 \omega_{M}\left(\tau_{k}\right) h_{k}=\widehat{H}\left(\boldsymbol{\xi}\left(\tau_{k}\right)\right)$ and that the function $\gamma(\boldsymbol{\xi})=\widehat{H}(\boldsymbol{\xi}) \max _{i \in\{1,2,3\}}\left|\xi_{i}\right| / 2$, is such that

$$
\begin{aligned}
V\left[\boldsymbol{\xi}\left(\tau_{k+1}\right)\right] & -V\left[\boldsymbol{\xi}\left(\tau_{k}\right)\right] \leqslant-\frac{15}{2} \omega_{M}\left(\tau_{k}\right) \xi_{M}\left(\tau_{k}\right) h_{k} \leqslant \\
& \leqslant-\frac{\widehat{H}(\boldsymbol{\xi})}{2} \max _{j \in\{1,2,3\}}\left|\xi_{j}\left(\tau_{k}\right)\right|=-\gamma\left(\boldsymbol{\xi}\left(\tau_{k}\right)\right),
\end{aligned}
$$

what concludes the proof.

Theorem 4.6 ensures that for system (11) it is possible to design a switching control scheme such that the closed loop system admits a function $V$ satisfying the hypotheses of Theorem 4.1 thus showing that the control scheme provides global asymptotic stability. In the next section the effectiveness of the scheme will be analyzed by showing the results of some simulations.

\subsection{Some comments on the rate of convergence}

The results presented above show that, theoretically, there exists a switching control scheme stabilizing the equilibrium $\boldsymbol{\omega}=\mathbf{0}, \boldsymbol{\xi}_{0}=(0,0,0,1)^{\top}$ of system (11). On the other hand, for the scheme to be applicable to a real system, some constraints needs to be fulfilled. In particular, the control signals are usually required to be bounded and approaching zero as the system approaches the equilibrium state; moreover, in the particular control scheme proposed herein, the time constant $h_{k}$ needs to have both an upperbound and a lowerbound. For, it is necessary and sufficient that it does not approach zero or infinity as $\boldsymbol{\xi}$ approaches $\boldsymbol{\xi}_{0}$. Now, from the definition of the switching strategy (Equation (6)) it is reasonably to suppose that $\xi_{1}\left(\tau_{k}\right), \xi_{2}\left(\tau_{k}\right)$ and $\xi_{3}\left(\tau_{k}\right)$ approach zero, as $k \rightarrow \infty$, with the same rate of convergence; say $\eta(k)$. Then $\left|\xi_{4}\right|-1=\sqrt{1-\xi_{1}^{2}-\xi_{2}^{2}-\xi_{3}^{2}}-1$ approaches zero with order $2 \eta(k)$. Moreover, $l\left(i, \boldsymbol{\xi}\left(\tau_{k}\right)\right)$ (Equation (19)) approaches zero with the same order of $-3\left(1-\xi_{i}\right)-1+\sqrt{9\left(1-3 \xi_{i}^{2}\right)+1+6\left(1-\xi_{i}\right)+3 \xi_{i}^{2}}$, i.e. with order $\eta(k)$, which means that $H\left(i, \boldsymbol{\xi}\left(\tau_{k}\right)\right)$ (see the definition $(20))$, approaches zero with order $2 \eta(k)$. On the other hand, from the definition of $\omega_{M}\left(\tau_{k}\right)$ (Equation (17)), it is clear that $\omega_{M}\left(\tau_{k}\right)$ cannot approach zero faster than $\bar{\omega}_{i}$ which, in turn, has order $\eta(k)$ (as seen from Equation (7)); in addition, the controls (15) are designed in such a way that $\omega_{M}\left(\tau_{k}\right)$ does not grows to infinity when both $\boldsymbol{\omega}_{P}$ and $\boldsymbol{\omega}_{D}$ approach zero. Hence $\omega_{M}\left(\tau_{k}\right)$ has the same order of $\bar{\omega}_{i}$, i.e. order $\eta(k)$. From these reasonings and from the definition of $h_{k}$, one can conclude that $h_{k} \rightarrow 0$ as $k \rightarrow \infty$, what is an undesirable behaviour.

In order to avoid this phenomenon, one can slightly change Equation (7) by assigning to $K$, instead of a constant value, a function of $\xi_{i}\left(\tau_{k}\right)$.

Finally, it can be proven that limiting the value of $h_{k}$ to a finite value, implies that the control signals do not grow to infinity and have the same order of $\xi_{i}\left(\tau_{k}\right)$ (see, for instance, the explicit expression in Example 4.1).

\section{Simulations}

The previous section has proven the existence of a switching control scheme which renders the equilibrium $\left(\mathbf{0}, \boldsymbol{\xi}_{0}\right)$ of (11) globally asymptotically stable.

Simulation have been performed to illustrate this behaviour, the results of which are plotted in Figures 3 and 4. They refer to an initial state of the angular rates $\boldsymbol{\omega}(0)=(1,0,0)^{\top}$, an initial attitude quaternion $\boldsymbol{\xi}(0) \simeq(0.7100,-0.1222,0.2550,0.6449)^{\top}$ (chosen randomly) and a constant value (equal to 0.01) for $h_{k}$.

In Figure 3 the time histories of $\xi_{1}, \xi_{2}$ and $\xi_{3}$ are plotted for the first 35 seconds. It can be noted that the convergence is reasonably fast. In Figure 4, instead, the time history of $V$ is depicted. A fast convergence towards zero is evident; nevertheless, as shown more in details in the small box, the function is not decreasing but only ANI. However, the overall convergence towards zero is guaranteed by Theorem 4.6.

\section{Conclusions}

A switching control scheme has been proposed and described in details to solve the global asymptotic stabilization problem for the attitude and the angular rates of an underactuated rigid body. In the first part of the paper only the kinematic equation has been considered and, supposing that the vector of angular rates is allowed 


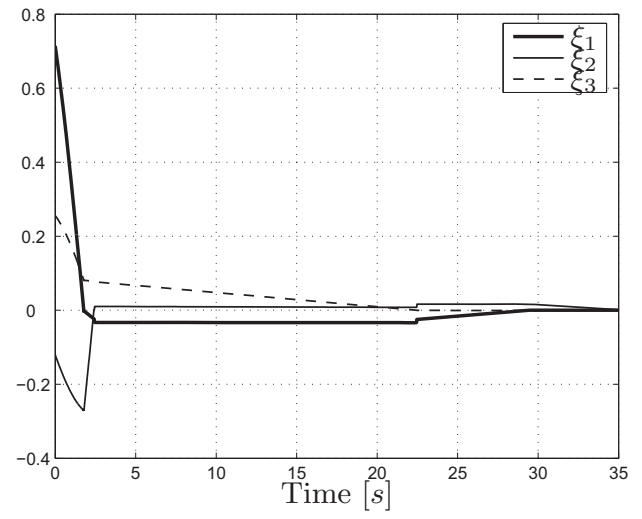

Fig. 3. Time behaviour of variables $\xi_{1}, \xi_{2}$ and $\xi_{3}$.

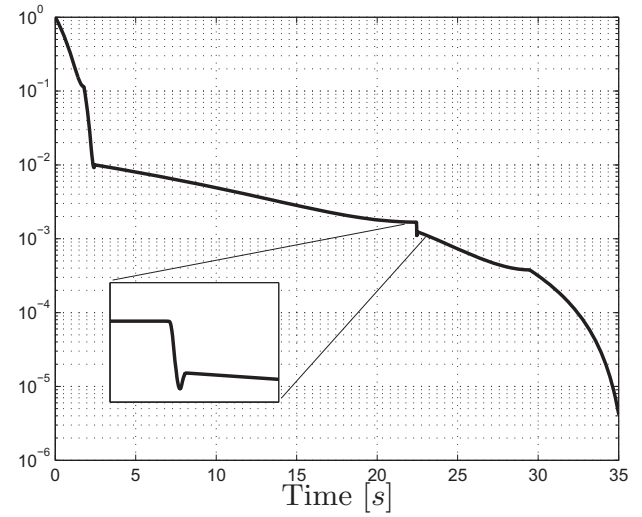

Fig. 4. Time behaviour of $V$.

to have a discontinuous behaviour, the proposed scheme has been proven to globally asymptotically stabilize the zero equilibrium. Then, the method has been extended to the whole system composed both by the kinematic and the dynamic equations. In this case a slight modification of the scheme formerly proposed in the paper has been proven to provide global asymptotic stability. The proof relies on a general stability theorem. Simulations are finally reported in order to show the effectiveness of the proposed methodology.

\section{References}

Aeyels, D., 1985. Stabilization by smooth feedback of the angular velocity of a rigid body. Systems \& Control Letters 5, 59-63.

Aeyels, D., Szafranski, M., 1988. Comments on the stabilizability of the angular velocity of a rigid body. Systems \& Control Letters 10, 35-39.

Astolfi, A., 1999. Output feedback stabilization of the angular velocity of a rigid body. Systems \& Control Letters 36, 181-192.

Branicky, M., 1998. Multiple Lyapunov functions and other analysis tools for switched and hybrid systems.
IEEE Transactions on Automatic Control 43 (4), 475482.

Brogliato, B., Niculescu, S.-I., Orhant, P., 1997. On the control of finite-dimensional mechanical systems with unilateral constraints. IEEE Transactions on Automatic Control 42 (2), 200-215.

Byrnes, C., Isidori, A., 1991. On the attitude stabilization of rigid spacecraft. Automatica (1), 87-95.

Coron, J., Keraï, E., 1996. Explicit feedbacks stabilizing the attitude of a rigid spacecraft with two control torques. Automatica 32 (5), 669-677.

Crouch, P., 1984. Spacecraft attitude control and stabilization: Applications of geometric control theory to rigid body models. IEEE Transactions on Automatic Control 29 (4), 321-331.

Goldstein, H., Poole, C., Safko, J., 2002. Classical Mechanics. Addison Wesley.

Isidori, A., 1995. Nonlinear Control Systems. Springer, London.

Krishnan, H., Reynahoglu, M., McClamroh, H., 1992. Attitude stabilization of a rigid spacecraft using gas jet actuators operating in a failure mode. In: Proceedings of the 31st Conference on Decision and Control. Tucson, Arizona, pp. 1612-1617.

Liberzon, D., 2003. Switching in Systems and Control. Birkhauser, Boston.

Lovera, M., Astolfi, A., 2004. Spacecraft attitude control using magnetic actuators. Automatica 40 (8), 14051414 .

Morin, P., Samson, C., Pomet, J., Jiang, Z., 1995. Timeverying feedback stabilization of the attitude of a rigid spacecraft with two controls. Systems \& Control Letters 25, 375-385.

Outbib, R., Sallet, G., 1992. Stabilizability of the angular velocity of a rigid body revisited. Systems \& Control Letters 18, 93-98.

Peleties, P., DeCarlo, R., 1991. Asymptotic stability of m-switched systems using lyapunov-like functions. In: Proceedings of the American Control Conference. Boston, USA, pp. 1679-1684.

Peleties, P., DeCarlo, R., 1992. Asymptotic stability of $\mathrm{m}$-switched systems using lyapunov functions. In: Proceedings of the IEEE Conference on Decision and Control. Tucson, Arizona, USA, pp. 3438-3439.

Sontag, E., Sussmann, H., 1988. Further comments on the stabilizability of the angular velocity of a rigid body. Systems \& Control Letters 12, 213-217.

Wen, J., Kreutz-Delgado, K., 1991. The attitude control problem. IEEE Transactions on Automatic Control 36 (10), 1148-1162.

Wertz, J., 1978. Spacecraft attitude determination and control. D. Reidel Publishing Company.

\section{Appendix}

Proof of Lemma 4.3. For $i=1$, Equation (3) yields $\dot{\xi}_{1}(t)=\left(\omega_{3}(t) \xi_{2}(t)-\omega_{2}(t) \xi_{3}(t)+\omega_{1}(t) \xi_{4}(t)\right) / 2$, which 
implies that for all $t \in\left[\tau_{k}, \tau_{k}+5 h_{k}\right]$,

$$
\begin{aligned}
\left|\dot{\xi}_{1}(t)\right| \leqslant \frac{1}{2}\left(\left|\omega_{3}(t) \xi_{2}(t)\right|\right. & +\left|\omega_{2}(t) \xi_{3}(t)\right|+ \\
& \left.+\left|\omega_{1}(t) \xi_{4}(t)\right|\right) \leqslant \frac{3}{2} \omega_{M}\left(\tau_{k}\right),
\end{aligned}
$$

where the bound $\left|\xi_{i}\right| \leqslant 1$ has been used. The reasoning can be repeated for $i \in\{2,3\}$ and hence the following inequalities hold for all $i\{1,2,3\}$

$$
-\frac{15}{2} \omega_{M}\left(\tau_{k}\right) h_{k} \leqslant \xi_{i}\left(\widetilde{\tau}_{k}\right)-\xi_{i}\left(\tau_{k}\right) \leqslant \frac{15}{2} \omega_{M}\left(\tau_{k}\right) h_{k},
$$

from which the thesis immediately follows.

Proof of Lemma 4.4. Observe that, as $\xi_{M}\left(\tau_{k}\right) \leqslant 1$ and considering the hypothesis on $h_{k}$,

$$
15 \omega_{M}\left(\tau_{k}\right) \xi_{M}\left(\tau_{k}\right) h_{k} \leqslant l\left(i, \tau_{k}\right)
$$

Consider, now, the equation $3 y^{2}+2\left[3\left|\xi_{4}\left(\tau_{k}\right)\right|+1\right] y-$ $\xi_{i}^{2}\left(\tau_{k}\right)=0$. Its two solutions have opposite sign, the positive one coinciding with $l\left(i, \tau_{k}\right)$. As a consequence, if $z$ is such that $0<z<l\left(i, \tau_{k}\right)$ then $3 z^{2}+2\left[3\left|\xi_{4}\left(\tau_{k}\right)\right|+1\right] z-\xi_{i}^{2}\left(\tau_{k}\right) \leqslant 0$. Hence, defining $r \triangleq 15 \omega_{M}\left(\tau_{k}\right) \xi_{M}\left(\tau_{k}\right) h_{k}$ and recalling Equation (A.2), $3 r^{2}+2\left[3\left|\xi_{4}\left(\tau_{k}\right)\right|+1\right] r-\xi_{i}^{2}\left(\tau_{k}\right) \leqslant 0$. Moreover, since $-r^{2} / \xi_{M}^{2}\left(\tau_{k}\right) \leqslant 0$ and $\left|\xi_{i}\left(\tau_{k}\right)\right| \leqslant \xi_{M}\left(\tau_{k}\right)$,

$$
\left[3-\frac{1}{\xi_{M}^{2}\left(\tau_{k}\right)}\right] r^{2}+2\left[3\left|\xi_{4}\left(\tau_{k}\right)\right|+\frac{\left|\xi_{i}\left(\tau_{k}\right)\right|}{\xi_{M}\left(\tau_{k}\right)}\right] r-\xi_{i}^{2}\left(\tau_{k}\right) \leqslant 0
$$

which can be rewritten as

$$
\left[\left|\xi_{4}\left(\tau_{k}\right)\right|-r\right]^{2}+\left[\left|\xi_{i}\left(\tau_{k}\right)\right|-\frac{r}{\xi_{M}\left(\tau_{k}\right)}\right]^{2} \geqslant\left[\left|\xi_{4}\left(\tau_{k}\right)\right|+2 r\right]^{2}
$$

Both terms in the previous inequality are positive; thus, the same inequality holds for their square roots:

$$
\sqrt{\left[\left|\xi_{4}\left(\tau_{k}\right)\right|-r\right]^{2}+\left[\left|\xi_{i}\left(\tau_{k}\right)\right|-\frac{r}{\xi_{M}\left(\tau_{k}\right)}\right]^{2}}>\left|\xi_{4}\left(\tau_{k}\right)\right|+2 r
$$

Now, note that, by the definition of $H\left(i, \boldsymbol{\xi}\left(\tau_{k}\right)\right)$, the hypothesis of Lemma 4.3 is fulfilled, and that the following two case are possible.

- If $\xi_{i}\left(\widetilde{\tau}_{k}\right) \geqslant 0$ then the first inequality in (A.1) can be written as $\left|\xi_{i}\left(\tau_{k}\right)\right|-15 \omega_{M}\left(\tau_{k}\right) h_{k} / 2 \leqslant\left|\xi_{i}\left(\widetilde{\tau}_{k}\right)\right|$.

- If $\xi_{i}\left(\widetilde{\tau}_{k}\right) \leqslant 0$ then the second inequality in (A.1) can be written as $-\left|\xi_{i}\left(\widetilde{\tau}_{k}\right)\right| \leqslant-\left|\xi_{i}\left(\tau_{k}\right)\right|+15 \omega_{M}\left(\tau_{k}\right) h_{k} / 2$, or equivalently, as $\left|\xi_{i}\left(\widetilde{\tau}_{k}\right)\right| \geqslant\left|\xi_{i}\left(\tau_{k}\right)\right|-15 \omega_{M}\left(\tau_{k}\right) h_{k} / 2$.

In both cases $\left|\xi_{i}\left(\widetilde{\tau}_{k}\right)\right| \geqslant\left|\xi_{i}\left(\tau_{k}\right)\right|-r /\left(2 \xi_{M}\left(\tau_{k}\right)\right)>$ $\left|\xi_{i}\left(\tau_{k}\right)\right|-r /\left(\xi_{M}\left(\tau_{k}\right)\right)>0$, the last inequality coming from the definitions of $r$ and $H\left(i, \boldsymbol{\xi}\left(\tau_{k}\right)\right)$. Moreover, from Equation (3) it is easy to compute (repeat, for $t=\widetilde{\tau}_{k}$, the reasoning yielding to Equation (23))

$$
\left|\xi_{4}\left(\tau_{k}\right)-\xi_{4}\left(\widetilde{\tau}_{k}\right)\right| \leqslant r / 2
$$

which yields ${ }^{5}\left|\xi_{4}\left(\widetilde{\tau}_{k}\right)\right| \geqslant\left|\xi_{4}\left(\tau_{k}\right)\right|-r / 2 \geqslant\left|\xi_{4}\left(\tau_{k}\right)\right|-r>0$, where, again, the last inequality comes from the definitions of $r$ and $H(i, \boldsymbol{\xi})$. Hence

$$
\sqrt{\rho\left(\widetilde{\tau}_{k}\right)} \geqslant \sqrt{\left(\left|\xi_{4}\left(\tau_{k}\right)\right|-r\right)^{2}+\left(\left|\xi_{i}\left(\tau_{k}\right)\right|-r / \xi_{M}\left(\tau_{k}\right)\right)^{2}} .
$$

Finally, from $(\text { A.4 })^{6},-\xi_{4}\left(\widetilde{\tau}_{k}\right) \geqslant-\left|\xi_{4}\left(\widetilde{\tau}_{k}\right)\right| \geqslant-\left[\left|\xi_{4}\left(\tau_{k}\right)\right|+\right.$ $r / 2]>-\left[\left|\xi_{4}\left(\tau_{k}\right)\right|+r\right]$. Hence, recalling Equation (A.3), one obtains $\sqrt{\rho\left(\widetilde{\tau}_{k}\right)}-\xi_{4}\left(\widetilde{\tau}_{k}\right)>r$ from which the thesis easily follows.
${ }^{5}$ For all $a, b \in \mathbb{R}^{+},|a+b| \leqslant|a|+|b|$ which, defining $c \triangleq a+b$, yields $|c|-|b| \leqslant|c-b|$.

${ }^{6}$ From footnote $5,|c|-|b| \leqslant|b-c|$, i.e. $\left|\xi_{4}\left(\widetilde{\tau}_{k}\right)\right|-\left|\xi_{4}\left(\tau_{k}\right)\right| \leqslant$ $\left|\xi_{4}\left(\tau_{k}\right)-\xi_{4}\left(\widetilde{\tau}_{k}\right)\right| \leqslant \frac{r}{2}$. 